\title{
Histological complete response with nivolumab for renal cell carcinoma with multiple metastases: A case report
}

\author{
SUGURU SHIROTAKE $^{1}$, GO KANEKO ${ }^{1}$, KOJI NAGATA ${ }^{2}$, MASAFUMI OYAMA ${ }^{1}$ and KOSHIRO NISHIMOTO ${ }^{1}$ \\ Departments of ${ }^{1}$ Uro-Oncology and ${ }^{2}$ Diagnostic Pathology, \\ Saitama Medical University International Medical Center, Saitama 350-1298, Japan
}

Received June 7, 2018; Accepted October 5, 2018

DOI: $10.3892 / \operatorname{mco} .2018 .1779$

\begin{abstract}
The present case report describes a case of left renal clear cell carcinoma with brain, lung, para-aortic, and lymph node metastases (cT1bN1M1) in a 52-year-old Japanese male. The patient received sequential anticancer treatments with pazopanib, everolimus, and axitinib, but exhibited treatment-resistant tumor growth. Treatment with nivolumab resulted in a complete response in metastatic sites. However, the residual renal tumor, which was enhanced by contrast medium, required radical nephrectomy. Pathological analyses of the renal tumor revealed that it consisted of fibrotic and lymphocyte-infiltrated tissues in which morphological cancer cells were not detected. The majority of lymphocytes were cluster of differentiation (CD)8-positive, suggesting that cancer cells were attacked by these lymphocytes. Retrospective analyses of renal cell carcinoma tissues, which were biopsied before the anticancer treatment, revealed their infiltration by CD8-positive T cells. To the best of our knowledge, this is the first case report to examine renal tissue prior to and following treatment with nivolumab using immunohistochemical analysis.
\end{abstract}

\section{Introduction}

Treatments using nivolumab, a humanized antibody targeting programmed cell death protein 1 (PD1), achieve durable effects in approximately $15 \%$ of patients with metastatic renal cell carcinoma (mRCC) (1-4). Choueiri et al (5) reported immunomodulatory activity of nivolumab in biopsied mRCC tissues, which were acquired before and in the course of treatment with nivolumab. However, a comparison study has not

Correspondence to: Dr Suguru Shirotake or Dr Koshiro Nishimoto, Department of Uro-Oncology, Saitama Medical University International Medical Center, 1397-1 Yamane, Hidaka, Saitama 350-1298, Japan

E-mail: ss197816@saitama-med.ac.jp

E-mail:knishi@saitama-med.ac.jp

Key words: metastatic renal cell carcinoma, nivolumab, complete response, cluster of differentiation 8-positive $\mathrm{T}$ cells, immunomodulatory activity yet been conducted on RCC tissue before and after treatment with nivolumab with a focus on infiltrating $\mathrm{T}$ cells. We herein describe a case of $\mathrm{mRCC}$ in which a complete response was achieved with nivolumab, present a detailed clinical course and pathological findings using $\mathrm{T}$ cell markers, and conduct a literature review.

\section{Case report}

Initial diagnosis. The present case was a 52-year-old Japanese male with left homonymous hemianopia. Computed tomography $(\mathrm{CT})$ and magnetic resonance imaging (MRI) revealed a left renal tumor, multiple lung nodules, and a metastatic brain tumor. He was referred to the Saitama Medical University International Medical Center (SIMC) for further examination and treatment (SIMC-Uro no. 8830, a unique non-sequential patient control number in the Department of Uro-Oncology, SIMC). Physical and laboratory examinations were normal, except for microhematuria. No previous history was reported. CT imaging (renal tumor, para-aortic lymph node, and lung metastases) and MRI (brain) during clinical course are shown as Fig. S1 (doi.org/10.6084/m9.figshare.7063880.v1), and Fig. S2 (doi.org/10.6084/m9.figshare.7063922.v1), respectively, and Fig. 1. CT (CT \#1; Fig. S1, 8 days before the initiation of the anticancer agent: Day \#-8) showed a left renal tumor $[85.8 \pm 0.9 \mathrm{~mm}$ (mean $\pm \mathrm{SEM})]$, an enlarged para-aortic lymph node $(9.9 \pm 0.1 \mathrm{~mm})$, and two left lung nodules (left \#1: $42.2 \pm 1.3 \mathrm{~mm}$. left \#2: 17.0 $\pm 0.6 \mathrm{~mm})$. All lesions were independently measured by TO and HK (Department of Uro-Oncology, Saitama Medical University International Medical Center), and TM (Department of Urology, Keio University School of Medicine), as previously reported (6). CT (CT\#1; Fig. S1) showed that the renal tumor was intensely enhanced by contrast medium soon after infusion and the enhancement was not observed in the delayed phase, suggesting that the tumor was clear cell carcinoma. MRI confirmed the brain tumor in the right occipital lobe $(18.5 \pm 0.7 \mathrm{~mm}, \mathrm{MRI} \# 1$; Fig. S2A). The patient underwent ultrasonography (US)-guided renal tumor biopsy and was pathologically diagnosed with clear cell carcinoma (Fig. 2K and $\mathrm{O}$ ).

Molecular targeted therapy using pazopanib (1st), everolimus (2nd), and axitinib (3rd). Pazopanib was initiated as first-line therapy ('paz' in Fig. 1A). In order to locally 
treat brain metastasis, the administration of pazopanib was temporarily stopped 9 days after its initiation (on Day \#9), and stereotactic radiotherapy was performed using the Cyber Knife G3 machine (Accuray Inc., Sunnyvale, CA, USA) on Days \#10, 11 and 13 (total 35 Gy, 'ck' in Fig. 1A). The administration of pazopanib was resumed on Day \#18. The brain metastasis gradually shrunk to $8.6 \pm 0.2 \mathrm{~mm}$ on Day \#58 (MRI \#2; Fig. S2B), to $1.6 \pm 1.6 \mathrm{~mm}$ on Day \#154 (MRI \#3; Fig. S2C), and disappeared after Day \#422 (MRI \#4-5; Fig. S2D and E). The complete response to treatment observed for brain metastasis can presumably be attributed to radiotherapy and/or pazopanib ('brain' in Fig. 1A). Similarly, the two lung lesions disappeared under pazopanib treatment ('lt. lung 1' and 'lt. lung 2' in Fig. 1A, respectively; 3rd and 5 th columns of Fig. S1, respectively). The renal tumor and para-aortic lymph node continued to shrink during the course of the treatment with pazopanib ('renal tumor' and 'para Ao' in Fig. 1A, respectively). De novo lung metastasis was detected in the lower lobe of the right lung ('rt. lung' in Fig. 1A; 4th column of Fig. S1). Therefore, the molecular targeted agent was altered from pazopanib to everolimus on Day \#335. The sizes of the renal tumor, para-aortic lymph node, and right lung metastasis did not significantly decrease between CT \#5 (Day \#307) and CT \#7 (Day \#462) (P>0.05, unpaired Student's t-test); therefore, everolimus was switched to axitinib. The treatment with axitinib was not effective because the sizes of the renal tumor and right lung metastasis increased from CT \#7 (Day \#462) to CT \#8 (Day \#539) ( $\mathrm{P}=0.0062$ and 0.0051 , respectively) (Fig. $1 \mathrm{~A}$ and $\mathrm{S} 1$ ). Four metastatic lesions (the brain, lt. lung \#1, lt. lung \#2, and para-aortic lymph node in Fig. 1A and S1) were controlled by the treatments described above, while de novo metastasis in the right lung (Fig. 1B) and the renal tumor with apparent contrast enhancement (Fig. 1C) required further anticancer treatment.

Immunotherapy using nivolumab (4th). A bi-weekly treatment with $207 \mathrm{mg}$ ( $3 \mathrm{mg} / \mathrm{kg}$ ) of nivolumab, which was approved for the treatment of $\mathrm{mRCC}$ in Japan at around that time, was initiated on Day \#563. After 5 courses of the treatment with nivolumab, de novo lung metastasis markedly decreased in size [from 20.4 $\pm 1.1 \mathrm{~mm}$ (CT \#8; Fig. 1C and S1) to $6.2 \pm 0.6 \mathrm{~mm}$ (CT \#9; Fig. 1E and S1), $\mathrm{P}=0.0003$ ]. The size of the unenhanced portion of the renal tumor remained unchanged ( ${ }^{*}$ in Fig. 1C and E), suggesting that it was necrotic tissue before treatment with nivolumab. The portion enhanced by contrast medium significantly contracted to a small mass with weak enhancement (pink arrowheads in Fig. 1C and E), suggesting that the primary lesion contained a small number of viable cancer cells.

Radical nephrectomy. The patient underwent left laparoscopic nephrectomy on Day \#728 without perioperative complications; however, it was not possible to remove the para-aortic lymph node. The pathological findings described below revealed no viable tumor cells, leading to the diagnosis of a pathological complete response. The patient resumed treatment with nivolumab because we are not able to clearly estimate the timing for discontinuation, and neither recurrence nor de novo metastasis including para-aortic lymph node was detected on Day \#836 (Fig. 1A).
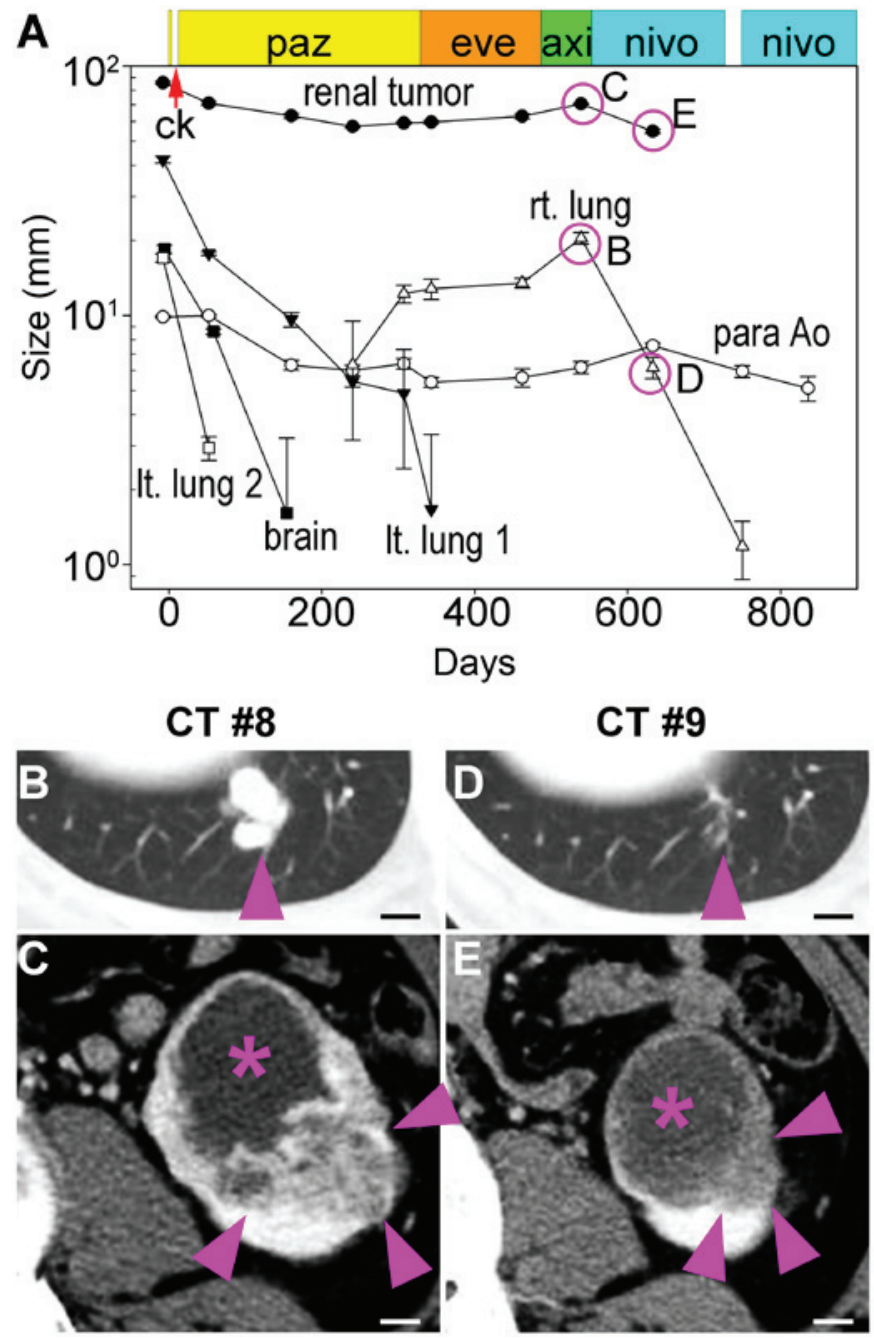

Figure 1. Clinical course of the case and $\mathrm{CT}$ images of representative lesions. (A) Clinical course of the case. The x-axes and y-axes of the graph indicate days following the initiation of the anticancer agent (Days) and the sizes of the six measurable lesions [renal tumor, brain metastasis (brain), left lung metastasis \#1 (lt. lung 1), left lung metastasis \#2 (lt. lung 2), right lung metastasis (rt. lung), and para-aortic lymph node metastasis (para Ao)]. These lesions were measured by three clinicians and the average value and standard errors are shown in the graph. Representative CT images [pink circles in panel (A)] prior to CT \#8, Day \#539 and following CT \#9, Day \#633 nivolumab treatment are shown in panels (B) (right lung metastasis, pink arrowhead) and (C) renal tumor, arrowheads and asterisks $\left(^{*}\right)$ indicate putative viable carcinoma tissue and a non-viable cystic lesion, respectively, and (D) right lung metastasis in $\mathrm{CT} \# 9$, the arrowhead indicates the corresponding portion of the right lung metastasis in panel (B). (E) Renal tumor, asterisks $\left(^{*}\right)$ and arrowheads indicate the corresponding portions of the asterisks $\left(^{*}\right)$ and the arrowhead in panel $(\mathrm{C})$, respectively. Yellow, orange, green, and blue rectangles indicate the days of treatment using pazopanib (paz, Days \#1-8 and Days \#18-334), everolimus (eve, Days \#335-495), axitinib (axi, Days \#496-562), and nivolumab (nivo, Days \#563-703 and Day \#750-836), respectively. CT, computed tomography; lt, left; rt, right; paz, pazopanib; eve, everolimus; axi, axitinib; nivo, nivolumab.

Pathological findings. The tumor was whitish with brown central necrosis in a coronal section of the extracted kidney (green arrowheads in Fig. 2A and B). A normal portion in block \#4 (one of the large number of tissue blocks prepared for a pathological diagnosis, ${ }^{*}$ in the left side of Fig. 2B) was histologically normal ( ${ }^{*}$ in Fig. 2C). Cancer cells were not detected in the tumor, into which a large number of CD8-positive $\mathrm{T}$ cells had infiltrated $\left({ }^{* *}\right.$ in Fig. $2 \mathrm{C}$ and D). 

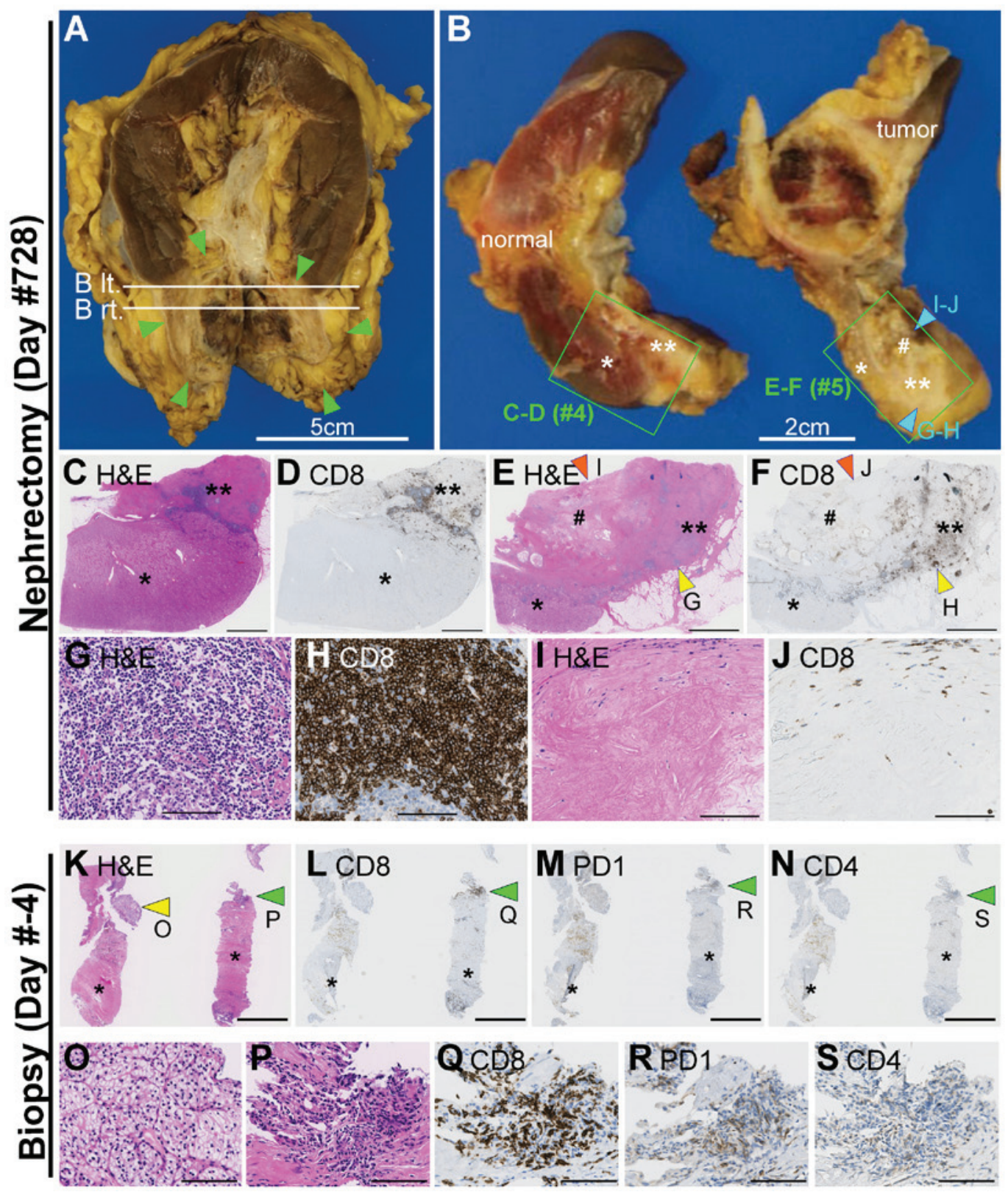

Figure 2. Pathological analyses. (A) Macroscopic image of a coronal section of the extracted kidney. Green arrowheads indicate the renal tumor. (B) Two cut surfaces of panel (A). Left and right cut surfaces correspond to the lines 'B lt.' and 'B rt.' in panel (A), respectively; 'normal' and 'tumor' indicate the normal and renal tumor portions, respectively. Blocks \#4 and \#5 were prepared from these tissues and portions are indicated by green frames in the left and right tissues, respectively. (C and D) Low magnification images of (C) H\&E and (D) immunostaining for CD8 in block \#4, respectively. (E and F) Low magnification images of (E) H\&E and (F) immunostaining for CD8 in block \#5, respectively. Scale bars in panels C-F indicate $5 \mathrm{~mm} .{ }^{*},{ }^{* *}$ and \# in panels (C-F) indicate normal renal tissue, renal tumor tissue, and fibrotic tissue, respectively. " and ${ }^{* *}$ in panels (C and D) correspond to those in the left-hand tissue of panel (B). ", ${ }^{* *}$ and \# in panels (E and F) correspond to those in the right-hand tissue of panel (B). (G and H) High magnification images of portions indicated by yellow arrowheads in panels (E and F), respectively (renal tumor portion in which a large number of CD8-positive T cells had infiltrated). (I and J) High magnification images of portions indicated by orange arrowheads in panels ( $\mathrm{E}$ and $\mathrm{F}$ ), respectively (fibrotic tissue in which a few $\mathrm{T}$ cells had infiltrated). Scale bars in panels (G-J) indicate $100 \mu \mathrm{m}$. (K) Low magnification images of H\&E staining of biopsied specimens. (L-N) Immunohistochemical staining images for CD8, PD1 and CD4 using serial sections of the coronal section in panel (K). Scale bars in panels (K-N) indicate 1 mm. * and arrowheads (yellow and green) indicate degenerated renal tissue and renal carcinoma tissue, respectively. (O-S) High magnification images of the portion indicated by the yellow and green arrowheads in panels (K-N), respectively. Scale bars in panels (O-S) indicate $100 \mu \mathrm{m}$. H\&E, hematoxylin and eosin; CD, cluster of differentiation; lt, left; rt, right; PD1, programmed cell death protein 1.

The immunohistochemical staining shown in Fig. 2D was performed using a mouse monoclonal anti human CD8 antibody (clone C8/144B; Dako, Santa Clara, CA, USA). Another tissue block (block \#5 in the right side of Fig. 2B) showed similar findings in normal tissue ( ${ }^{*}$ in Fig. 2E and F) and tumor tissue $\left[^{* *}\right.$ in Fig. $2 \mathrm{E}$ and $\mathrm{F}$, and $2 \mathrm{G}$ and $\mathrm{H}$ (enlarged image of the yellow arrowheads in Fig. 2E and F)] as previously reported (7). The inner tissue of the tumor was completely occupied by fibrotic tissue, into which only a few $\mathrm{T}$ cells had infiltrated [\# the right side of Fig. 2B, E and F, as well as Fig. 2I and $\mathbf{J}$ (enlarged image of the orange arrowheads in Fig. 2E and F)]. In summary, the pathological findings 
in this case exhibited the effects of nivolumab on RCC as follows: i) No impact on normal renal tissue (no T cell infiltration); ii) significant $\mathrm{T}$ cell infiltration into presumably viable tumor tissue ( ${ }^{* *}$ in Fig. 2B-F, 2G and H); and iii) fibrosis after presumable necrosis and/or phagocytosis after $\mathrm{T}$ cell infiltration induced by nivolumab (\# in Fig. 2B, E and F as well as Fig. 2I and J).

\section{Discussion}

In 2014, Tumeh et al (8) reported that melanoma tissues infiltrated with many CD8-positive $\mathrm{T}$ cells are associated with a good response to therapy using pembrolizumab, an immune checkpoint inhibitor targeting the PD-1, PD-L1 axis (termed adaptive immune resistance $(4,9)$. In these tissues, the number of CD8-positive T cells is significantly increased by pembrolizumab, leading to melanoma cell injury. Similar histological findings may be observed in RCC tissues before and after treatments using nivolumab; however, to our knowledge, a comparison of RCC tissues before and after treatment with nivolumab was not yet conducted.

We retrospectively analyzed biopsy specimens (on Day \#-4) obtained from the patient using the same anti-CD8 antibody, mouse monoclonal anti-human PD1 antibody (NAT105; Abcam, Tokyo, Japan), and mouse monoclonal anti-human CD4 antibody (clone 1F6; Novocastra, Tokyo, Japan) (Fig. 2K-N). The specimen consisted of degenerated non-cancer renal tissues ( ${ }^{*}$ in Fig. $2 \mathrm{~K}$ ) and clear cell carcinoma tissues (yellow and green arrowheads). Carcinoma tissues were infiltrated by a large number of T cells (Fig. 2P), which expressed CD8 (Fig. 2L), PD1 (Fig. 2M), and CD4 (Fig. 2N) at different levels. Fig. 2P-S show the carcinoma tissue portion that expressed CD8 at the highest level within the biopsy specimen. Even in that portion (Fig. 2Q), the number of CD8-positive $\mathrm{T}$ cells was markedly lower than in the tumor portion of nephrectomized tissue (Fig. $2 \mathrm{H}$ ). T cells in the tumor portion more weakly expressed PD1, suggesting that most PD1-positive carcinoma cells were attacked by a higher number of CD8-expressing T cells. The clinical course and pathological findings described above imply that CD8 immunohistochemistry for biopsied specimens of RCC tissue may potentially predict the effectiveness of nivolumab, as reported previously by Tumeh et al (8) in melanoma tissues.

We herein report a case of $\mathrm{mRCC}$ that achieved a complete response with nivolumab using detailed histochemical (H\&E) and immunohistochemical analyses. The number of CD8-expressing $\mathrm{T}$ cells in biopsied specimens may play a role in predicting the treatment effects of nivolumab, as previously reported for pembrolizumab in melanoma tissue. Further investigations are needed in order to confirm this hypothesis from a single case experiment.

This is the first case report to show that nivolumab contributed to achieving a histological as well as radiological complete response in a patient with multiple metastatic RCC who had not responded to other molecular targeted therapies.

\section{Acknowledgements}

The authors would like to thank Dr Tsugio Seki (California University of Science and Medicine and Saitama Medical
University International Medical Center) for reviewing the manuscript and for financial support for publication, and also Taiki Hayashi, Takashi Okabe, Hideyuki Kondo (Department of Uro-Oncology, Saitama Medical University International Medical Center) and Tsukasa Masuda (Department of Urology, Keio University School of Medicine) for collecting the patient's data.

\section{Funding}

The present study was supported in part by a JSPS Grant-in-Aid for Scientific Research (JP17K18062), and also by Dr Tsugio Seki (California University of Science and Medicine and Saitama Medical University International Medical Center).

\section{Availability of data and materials}

Figures $\mathrm{S} 1$ and $\mathrm{S} 2$ are available in the Figshare repository. Figure S1, CT imaging during clinical course: doi.org/10.6084/ m9.figshare.7063880.v1; Figure S2, MRI showing brain metastasis during clinical course: doi.org/10.6084/m9.figshare. 7063922.v1.

\section{Authors' contributions}

SS and GK planned the case study, drafted the manuscript, and analyzed the patient data. KojN performed immunohistochemical examination and interpreted the pathological findings. MO and KosN accurately interpreted the case study and critically revised this manuscript for important intellectual content. All authors read and approved the version to be published.

\section{Ethics approval and consent to participate}

Not applicable.

\section{Patient consent for publication}

The patient provided written informed consent for the publication of this case report.

\section{Competing interests}

The authors declare that they have no competing interests.

\section{References}

1. McDermott DF, Drake CG, Sznol M, Choueiri TK, Powderly JD, Smith DC, Brahmer JR, Carvajal RD, Hammers HJ, Puzanov I, et al: Survival, durable response, and long-term safety in patients with previously treated advanced renal cell carcinoma receiving nivolumab. J Clin Oncol 33: 2013-2020, 2015.

2. Motzer RJ, Escudier B, McDermott DF, George S, Hammers HJ Srinivas S, Tykodi SS, Sosman JA, Procopio G, Plimack ER, et al; CheckMate 025 investigators: Nivolumab versus everolimus in advanced renal-cell carcinoma. N Engl J Med 373: 1803-1813, 2015.

3. Hsieh JJ, Purdue MP, Signoretti S, Swanton C, Albiges L, Schmidinger M, Heng DY, Larkin J and Ficarra V: Renal cell carcinoma. Nat Rev Dis Primers 3: 17009, 2017.

4. Pardoll DM: The blockade of immune checkpoints in cancer immunotherapy. Nat Rev Cancer 12: 252-264, 2012. 
5. Choueiri TK, Fishman MN, Escudier B, McDermott DF, Drake CG, Kluger H, Stadler WM, Perez-Gracia JL, McNeel DG, Curti B, et al: Immunomodulatory activity of nivolumab in metastatic renal cell carcinoma. Clin Cancer Res 22: 5461-5471, 2016

6. Kondo H, Shirotake S, Okabe T, Makino S, Nishimoto K and Oyama M: Clinical impact of consolidative and salvage radiotherapy for lymph node metastasis in upper urinary tract urothelial carcinoma. Case Rep Urol 2018: 1471839, 2018.

7. Makita K, Nishimoto K, Kiriyama-Kitamoto K, Karashima S, Seki T, Yasuda M, Matsui S, Omura M and Nishikawa T: A novel method: Super-selective adrenal venous sampling. J Vis Exp: Sep 15, 2017 (Epub ahead of print).
8. Tumeh PC, Harview CL, Yearley JH, Shintaku IP, Taylor EJ, Robert L, Chmielowski B, Spasic M, Henry G, Ciobanu V, et al: PD-1 blockade induces responses by inhibiting adaptive immune resistance. Nature 515: 568-571, 2014.

9. Spranger S, Spaapen RM, Zha Y, Williams J, Meng Y, Ha TT and Gajewski TF: Up-regulation of PD-L1, IDO, and T(regs) in the melanoma tumor microenvironment is driven by CD8(+) T cells. Sci Transl Med 5: 200ra116, 2013. 